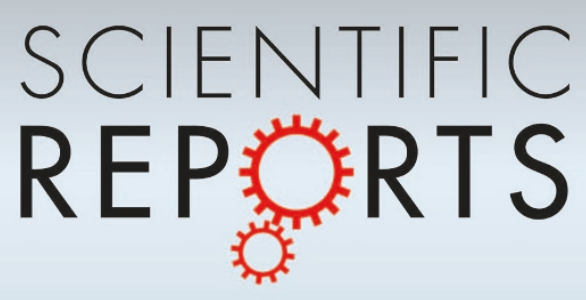

\title{
Scalable and Direct Growth of Graphene Micro Ribbons on Dielectric Substrates
}

SUBJECT AREAS:

ELECTRONIC DEVICES

SYNTHESIS OF GRAPHENE

ELECTRONIC PROPERTIES AND

DEVICES

SYNTHESIS AND PROCESSING

Received

30 November 2012

Accepted

15 February 2013

Published

27 February 2013

Correspondence and requests for materials should be addressed to T.-L.R. (rentl@tsinghua. edu.cn) or Y.G.Z. lygzhang2012@ sinano.ac.cn)

* These authors contributed equally to this work.

\author{
Debin Wang * , He Tian ${ }^{1,2,3 *}$, Yi Yang ${ }^{2,3}$, Dan Xie ${ }^{2,3}$, Tian-Ling Ren ${ }^{2,3} \&$ Yuegang Zhang ${ }^{1,4}$
}

\begin{abstract}
'Materials Sciences Division and the Molecular Foundry, Lawrence Berkeley National Laboratory, Berkeley, California 94720, United States, ${ }^{2}$ Institute of Microelectronics, Tsinghua University, Beijing 100084, China, ${ }^{3}$ Tsinghua National Laboratory for Information Science and Technology (TNList), Tsinghua University, Beijing 100084, China, ${ }^{4}$ Suzhou Institute of Nano-Tech and Nano-Bionics, Chinese Academy of Sciences, Suzhou 215123, China.
\end{abstract}

Here we report on a scalable and direct growth of graphene micro ribbons on $\mathrm{SiO}_{2}$ dielectric substrates using a low temperature chemical vapor deposition. Due to the fast annealing at low temperature and dewetting of $\mathrm{Ni}$, continuous few-layer graphene micro ribbons grow directly on bare dielectric substrates through $\mathrm{Ni}$ assisted catalytic decomposition of hydrocarbon precursors. These high quality graphene micro ribbons exhibit low sheet resistance of $\sim 700 \Omega-2100 \Omega$, high on/off current ratio of $\sim 3$, and high carrier mobility of $\sim 655 \mathrm{~cm}^{2} \mathrm{~V}^{-1} \mathrm{~s}^{-1}$ at room temperature, all of which have shown significant improvement over other lithography patterned CVD graphene micro ribbons. This direct approach can in principle form graphene ribbons of any arbitrary sizes and geometries. It allows for a feasible methodology towards better integration with semiconductor materials for interconnect electronics and scalable production for graphene based electronic and optoelectronic applications where the electrical gating is the key enabling factor.

raphene is a fascinating two-dimensional carbon material. It has attracted great research interest owing to its unique electronic structures and appealing physical properties ${ }^{1,2}$. Its excellent transport properties, high carrier mobility, and the ability to tune the carrier concentration with electrical gates make it a material with great potential applications ranging from radio-frequency devices and transistors to optoelectronic devices $^{3,4}$. The broad optical IR absorption in graphene can be modulated through electrical gating ${ }^{5}$. Tunable tetrahertz plasmonic resonance has been also realized on engineered graphene micro ribbons ${ }^{6}$. Graphene has also been considered as a promising candidate for interconnect technology due to its excellent electrical performance. High frequency operation of graphene micro ribbon interconnects at $1.3 \mathrm{GHz}$ has been demonstrated on CMOS electronics ${ }^{7}$.

Large scale graphene films can be obtained from both physical routes and chemical routes. The mechanical cleavage of highly oriented pyrolytic graphite produces pristine graphene with very low concentration of defects $^{8,9}$. But the size, thickness and location of the graphene flakes are uncontrollable. Covalent and noncovalent exfoliation of graphite or graphite oxide in solution can introduce structural disorder ${ }^{10-12}$. Conversion of $\mathrm{SiC}$ to graphene through $\mathrm{Si}$ sublimation that can produce high quality wafer scale graphene ${ }^{13-15}$ usually comes with high cost. The most promising and low cost approach to produce good quality graphene is chemical vapor deposition (CVD) of hydrocarbon precursors onto transition metal substrates, such as $\mathrm{Ni}^{16-18}, \mathrm{Cu}^{19,20}, \mathrm{Pd}^{21}, \mathrm{Ru}^{22}$, $\mathrm{Ir}^{23}, \mathrm{Co}^{24}$, and $\mathrm{Pt}^{25}$. A judicial choice of transition metal can produce large scale and uniform monolayer graphene on $\mathrm{Cu}$, bi-layer or few-layer graphene on $\mathrm{Ni}$. It usually requires polymer assisted transfer and metal etching steps to place the CVD graphene films on to dielectric substrates. Recently certain transfer-free methodologies have been proposed ${ }^{18,20}$. Large scale graphene films can directly grow on dielectric substrates with no traces of polymer left from a transfer step. For practical device applications, however, lithography patterning techniques, such as optical lithography or electron beam lithography followed by lift off and plasma etching procedures, are required to tailor the graphene films into desired sizes and shapes for real device applications. Both the graphene transfer and the fabrication procedures always inevitably lead to deteriorated electrical and optical performances of graphene due to chemical contamination ${ }^{26,27}$ or structural damage ${ }^{1,28-30}$ during the etching and transferring processes. This has motivated a great research thrust on improving the quality of patterned graphene driven by the increasing need of band gap engineering ${ }^{14,31,32}$ and micrometer scale graphene interconnect wires for highspeed integrated circuits ${ }^{7,33}$.

Here we report on a scalable and direct growth of graphene micro ribbons on $\mathrm{SiO}_{2}$ dielectric substrates using a one-step chemical vapor deposition. Due to the efficient diffusion of carbon source through micro-/nano-grain boundaries along the periphery of pre-patterned $\mathrm{Ni}$ films and the dewetting of $\mathrm{Ni}$ on the dielectric substrate 

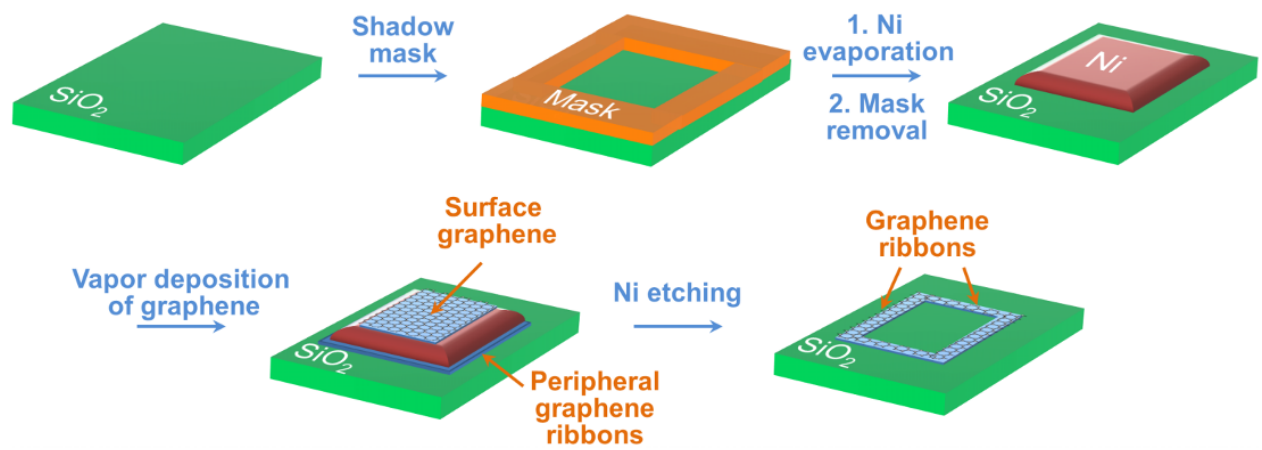

Figure 1 Schematics of the direct growth of graphene micro ribbons along the periphery of pre-patterned $\mathrm{Ni}_{\text {on }}$ a $\mathrm{SiO}_{2}$ dielectric substrate. $\mathrm{Ni}$ is first patterned on $\mathrm{SiO}_{2}$ substrate through thermal evaporation using a shadow mask. After a chemical vapor deposition, few-layer graphene films are found on top of the pre-patterned $\mathrm{Ni}$ film and continuous graphene micro ribbon is grown along the periphery of the Ni film. After $\mathrm{Ni}$ etching step, the patterned Ni film and surface graphene films on top are removed, leaving only graphene micro ribbons on the dielectric substrate, avoiding any chemical contamination and structural deterioration that usually come with polymer assisted transfer process and lithography patterning process followed by lift off and plasma etching.

during the chemical vapor deposition, continuous micro ribbons of few-layer graphene $(2-10 \mu \mathrm{m}$ in width and a few millimeters in length) grow directly on bare dielectric substrates. Scanning electron microscopy, energy-dispersive spectroscopy mapping, atomic force microscopy, and confocal Raman spectroscopy and mapping confirm the formation of the continuous graphene ribbons. The asgrown graphene micro ribbons exhibit excellent structural integrity and electrical properties.

\section{Results}

Our design of the direct growth of graphene micro ribbons is best illustrated in Fig. 1. In brief, we used thermal evaporation of $\mathrm{Ni}$ source to deposit $100 \mathrm{~nm}$ thick patterned $\mathrm{Ni}$ films on to the $\mathrm{SiO}_{2}$ substrate. The shape of the Ni films was defined by the shadow mask placed in close contact with the $\mathrm{SiO}_{2}$ substrate before the thermal evaporation. The samples were then placed inside a quartz tube in a CVD furnace to grow graphene. Hydrocarbon source of graphene
$\left(\mathrm{C}_{2} \mathrm{H}_{4}, 1 \mathrm{sccm}\right)$ was supplied into the quartz tube for annealing at $750^{\circ} \mathrm{C}$ for 5 minutes with protective and reductive gas flow $\left(\mathrm{H}_{2}: \mathrm{Ar}=\right.$ $50 \mathrm{sccm}: 150 \mathrm{sccm}$ ) under low pressure (10 Torr). The catalytic Ni film facilitates the decomposition of $\mathrm{C}_{2} \mathrm{H}_{4}{ }^{17}$. The high solubility of carbon in Ni promotes the highly efficient diffusion through the $\mathrm{Ni}$ grain boundaries. Graphene films were deposited on top of the $\mathrm{Ni}$ film, and continuous graphene micro ribbons were formed along the periphery of Ni films. Both the graphene films and micro ribbons were defined by the shadow mask. After Ni was etched away, graphene films grown on top were subsequently removed, leaving only graphene micro ribbons on the dielectric substrate, without any polymer assisted transferring, photolithography, and plasma etching steps.

The number of graphene layers and structural quality of as-grown graphene micro ribbon was examined by confocal Raman spectroscopy and microscopy, which is a powerful and well recognized method for structural characterization of carbon materials ${ }^{34,35}$.
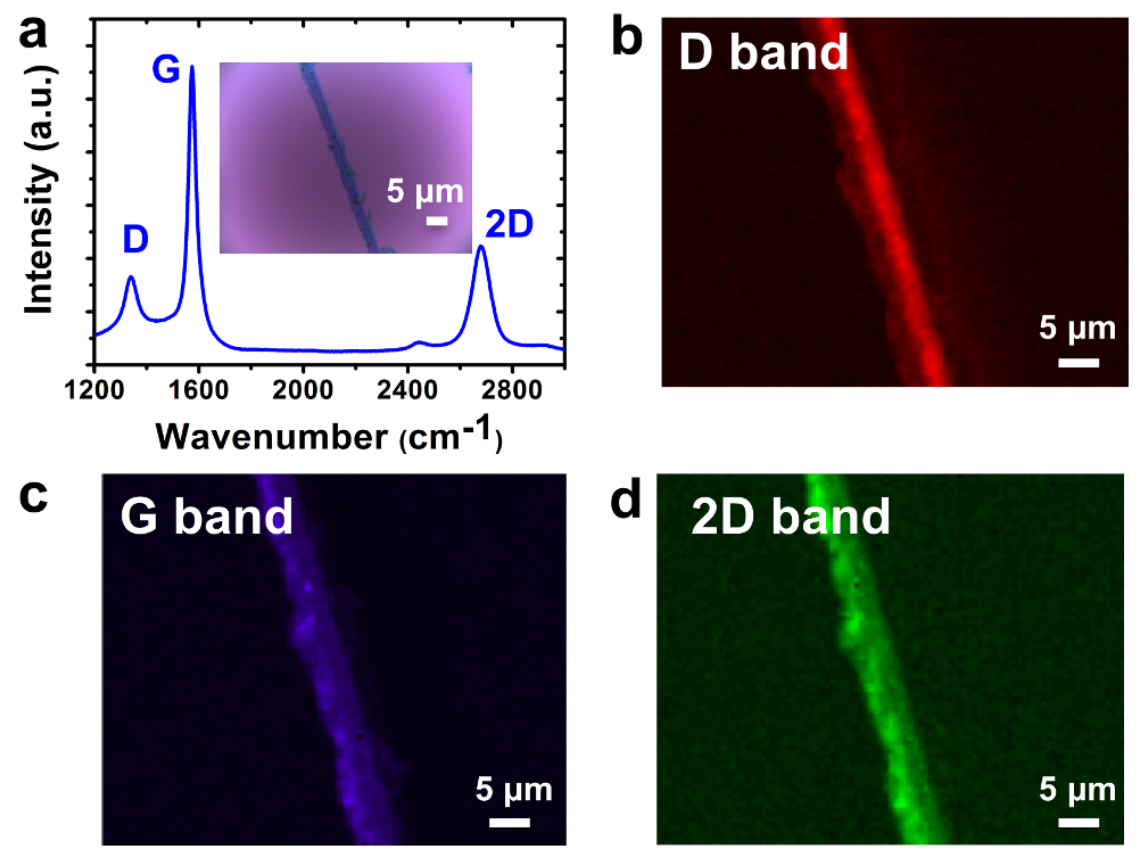

Figure $2 \mid$ Raman characterization of graphene micro ribbons. A typical Raman spectrum (a) and Raman mapping of D band, G band, and 2D band (b, c, and d) of a graphene micro ribbon ( $3 \mu \mathrm{m}$ wide, $50 \mu \mathrm{m}$ long, as shown in the optical image of the inset of (a)). The ratio of $2 \mathrm{D}$ band and $\mathrm{G}$ band $\mathrm{I}_{2 \mathrm{D}} / \mathrm{I}_{\mathrm{G}} \sim 0.40$ indicates the few-layer thickness of the graphene ribbon. A minimal structural disorder is observed by the ratio of $\mathrm{D}$ band and $\mathrm{G}$ band $\mathrm{I}_{\mathrm{D}} / \mathrm{I}_{\mathrm{G}} \sim 0.26$ 
Fig. 2 shows a typical Raman spectrum and mappings of D band, G band, and $2 \mathrm{D}$ band of a graphene ribbon $(3 \mu \mathrm{m}$ wide $50 \mu \mathrm{m}$ long) directly grown on $\mathrm{a} \mathrm{SiO}_{2}$. The spectrum (Fig. 2a) shows the $\mathrm{G}$ band peak at $\sim 1580 \mathrm{~cm}^{-1}$ and $2 \mathrm{D}$ band peak at $\sim 2700 \mathrm{~cm}^{-1}$. The full width at half maximum (FWHM) of the $2 \mathrm{D}$ band is $\sim 70 \mathrm{~cm}^{-1}$. The $2 \mathrm{D}$ to $\mathrm{G}$ peak ratio $\left(\mathrm{I}_{2 \mathrm{D}} / \mathrm{I}_{\mathrm{G}}\right)$ is about 0.40 . The position and FWHM of $2 \mathrm{D}$ band, along with $\mathrm{I}_{2 \mathrm{D}} / \mathrm{I}_{\mathrm{G}}$, indicate the micro ribbon is mostly a few layers (about 3-5 layers) in thickness ${ }^{34}$. The existence of $\mathrm{D}$ band at $\sim 1350 \mathrm{~cm}^{-1}$ suggests that this directly grown graphene micro ribbon has certain structural defects, inherent to most CVD synthesis of graphene. Prior few-layer or multi-layer CVD graphene grown on catalytic metal film usually shows the $D$ peak to $G$ peak ratio $\left(\mathrm{I}_{\mathrm{D}} / \mathrm{I}_{\mathrm{G}}\right)$ of $0.2-0.5^{17,20,36,37}$, largely due to the structural defects such as graphene edges and sub-domain boundaries ${ }^{34}$. Graphene ribbons obtained from exfoliation of HOPG always show lower $\mathrm{I}_{\mathrm{D}} / \mathrm{I}_{\mathrm{G}}$ values because of better structural integrity ${ }^{34,38}$. In our case, we found the $\mathrm{I}_{\mathrm{D}} / \mathrm{I}_{\mathrm{G}}$ ratio is about 0.26 , suggesting good quality fewlayer micro ribbons among those grown from CVD methods. The Raman mapping of the D band, G band, and 2D band (Fig. 2b, $2 c$ and 2d) show a continuous, few-layer thick graphene ribbon is formed along its entire length.

\section{a Before $\mathrm{Ni}$ etching}

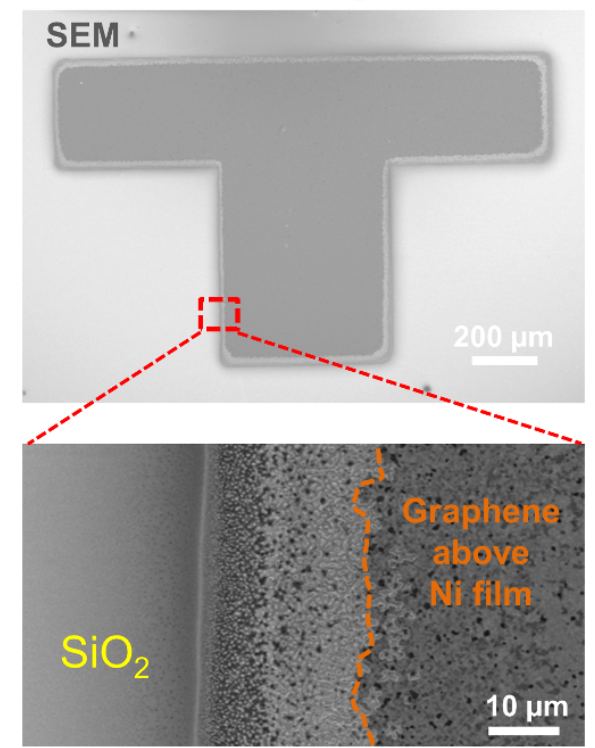

b

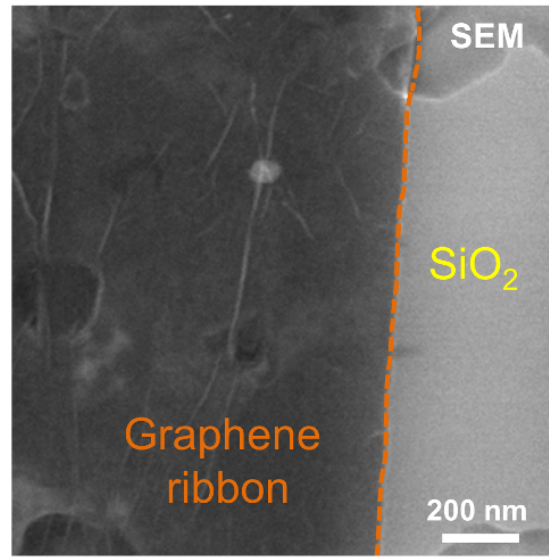

C
The morphology of the graphene micro ribbons was further characterized by scanning electron microscopy (SEM) and atomic force microscopy (AFM). In Fig. 3a, a "T" shape Ni film (120 nm thick) patterned by a shadow mask on a $\mathrm{SiO}_{2}$ substrate after the deposition of graphene is shown. Graphene films were deposited above the $\mathrm{Ni}$ film, and continuous graphene micro ribbons ( $\sim 5 \mu \mathrm{m}$ wide) were formed along the periphery of the $\mathrm{Ni}$ film. Both the graphene films and the micro ribbons are clearly seen in the SEM micrographs. The gray scale contrast in the SEM micrograph reveals the electrical conductivity contrast between the conducting graphene films and ribbons (dark contrast) and the insulating $\mathrm{SiO}_{2}$ substrate (bright contrast due to the electron charging). After the etching step, the Ni film template was removed along with the graphene film on top, leaving only a few millimeters long and continuous graphene microribbons on the dielectric substrate. A closer look at the graphene ribbons grown by this direct method reveals that the micro ribbons are firmly attached to the dielectric substrate where wrinkles are clearly visible (Fig. 3b). Unlike the mechanism found in conventional polymer assisted transferring methods ${ }^{28}$, the difference in thermal expansion coefficient between $\mathrm{Ni}$ and graphene inevitably leads to wrinkles in our transfer-free method ${ }^{39,40}$. This is also an indicator of

\section{After Ni etching}
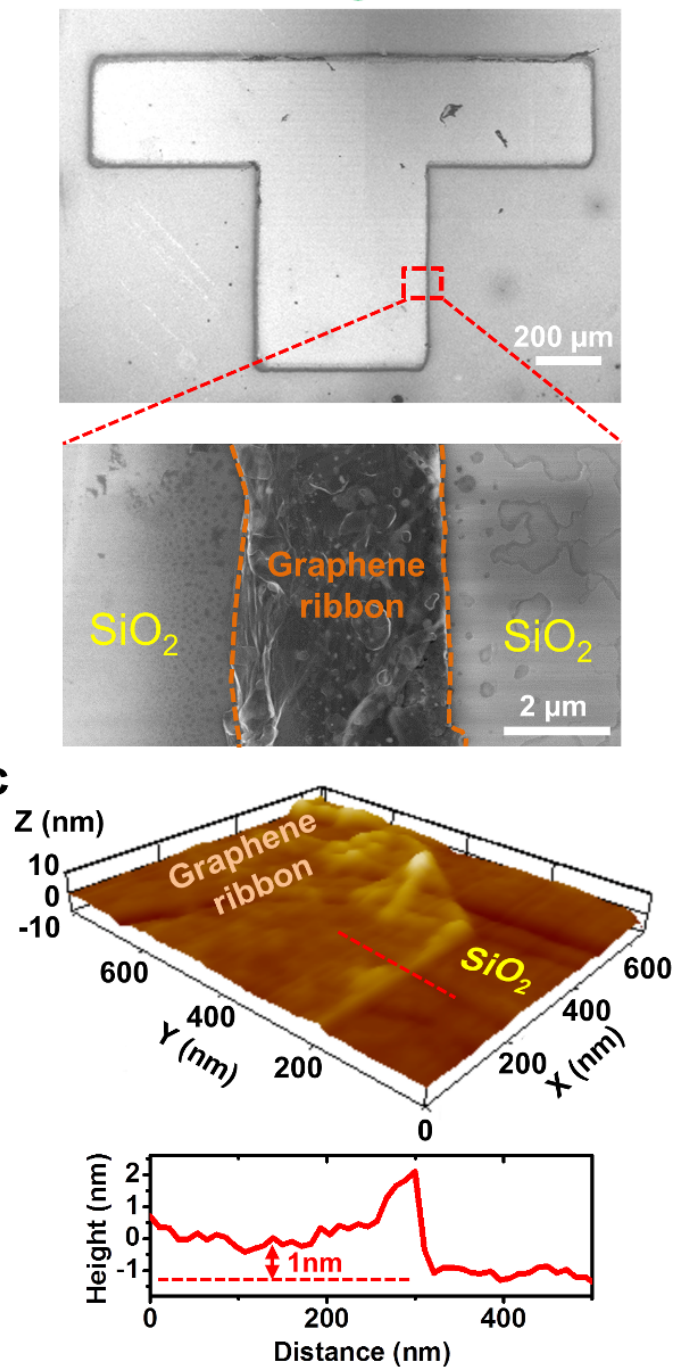

Figure 3 Few-layer graphene micro ribbons characterized by SEM microscopy and AFM. (a) The SEM micrograph reveals the formation of graphene micro ribbons along the periphery of a "T" shape Ni film patterned by a shadow mask on a $\mathrm{SiO}_{2}$ substrate. The graphene films deposited on top of the Ni film is etched away along with the underneath Ni film at the end of the etching step. (b) The SEM imaging reveals the wrinkles of the graphene micro ribbons caused by the mismatch of the thermal expansion coefficient between $\mathrm{Ni}$ and graphene. (c) AFM tapping mode 3D topography image of a partial graphene micro ribbon shows $\sim 1 \mathrm{~nm}$ thickness in the height profile along red dash line, confirming the number of graphene layers is about 3 . 

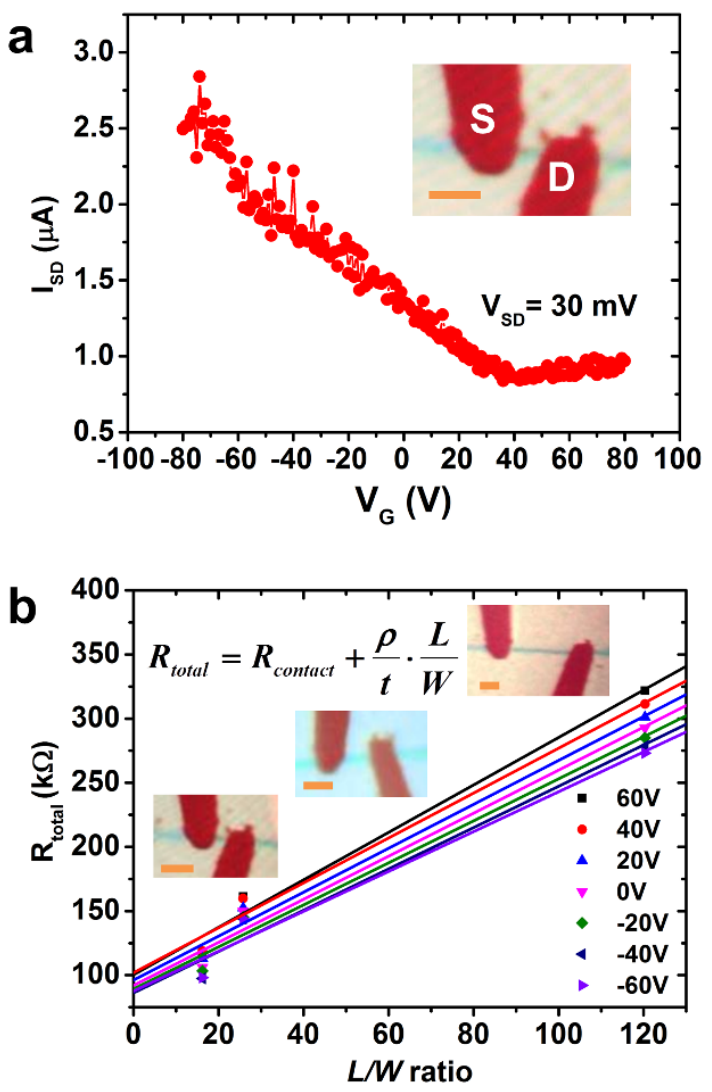

Figure 4 Electrical transport tests of graphene micro ribbons. A back gated two-point $\mathrm{I}_{\mathrm{SD}}-\mathrm{V}_{\mathrm{G}}$ characteristic curve on a graphene ribbon at room temperature under vacuum $\left(2 \times 10^{-3}\right.$ Torr $)$ is shown (a). The Dirac neutrality point was found to be at $\sim 40 \mathrm{~V}$. The conductance was modulated by a factor of $\sim 3$. The field effect mobility of hole and electron carriers at room temperature were extracted from the slope of the curve to be $\mu_{\mathrm{h}}=655 \mathrm{~cm}^{2} \mathrm{~V}^{-1} \mathrm{~s}^{-1}$, and $\mu_{\mathrm{e}}=111 \mathrm{~cm}^{2} \mathrm{~V}^{-1} \mathrm{~s}^{-1}$. Because tungsten electrodes have direct contact with the graphene ribbons in the transport tests, non-negligible electrical contact resistance is expected. The total electrical resistance, $R_{\text {total }}$, is plotted as a function of channel length to width ratio $(\mathrm{L} / \mathrm{W})$ under different gate biases $\left(\mathrm{V}_{\mathrm{G}}\right.$, between $+60 \mathrm{~V}$ and $-60 \mathrm{~V})$. The contact resistance, $\mathrm{R}_{\text {contact }}$, can be extracted from the linear fit (as shown in (b)) to be $R_{\text {contact }}=93 \pm 6 \mathrm{k} \Omega$. The sheet resistance, $R_{\text {sheet }}=\rho / t$, can be extracted from the slopes of the linear fit curves. The values decreased from $1850 \Omega$ per square to $1550 \Omega$ per square, with decreasing $\mathrm{V}_{\mathrm{G}}$ (from $+60 \mathrm{~V}$ to $-60 \mathrm{~V}$ accordingly). Scale bars: $50 \mu \mathrm{m}$.

structural defects and cracking at some local sites that lead to the disorder peak of Raman D band as shown in Fig. $2 \mathrm{a}^{34}$. High spatial resolution AFM topography imaging of a partial graphene ribbon (Fig. $3 \mathrm{c}$ ) revealed the thickness of the graphene ribbons to be $\sim 1 \mathrm{~nm}$. Given the spacing between each graphene lattice layer of $\sim 3.4 \AA^{41}$, the number of layers of the micro ribbons is about 3 . This is also consistent with the ratio of Raman $2 \mathrm{D}$ band to $\mathrm{G}$ band $\left(\mathrm{I}_{2 \mathrm{D}} / \mathrm{I}_{\mathrm{G}} \sim\right.$ 0.40 ) as shown in Fig. 2a.

The electrical properties of the as-grown graphene micro ribbons were evaluated with a back-gated field effect transistor layout. The graphene micro ribbons were readily grown on a $\mathrm{SiO}_{2}$ dielectric substrate. The samples under test were placed into a probe station. Two tungsten microprobes ( $25 \mu \mathrm{m}$ radius) were used as the source and the drain electrodes and placed directly at two ends of several selected segments of graphene micro ribbons. An Ohmic contact was established between tungsten probes and graphene micro ribbons (Fig. S2). The highly doped $\mathrm{Si}$ under the $\mathrm{SiO}_{2}$ dielectric layer was used as the gate electrode. The source-drain current $\left(\mathrm{I}_{\mathrm{SD}}\right)$ was modulated by sweeping a back gate voltage $\left(\mathrm{V}_{\mathrm{G}}\right)$ at room temperature and under vacuum condition $\left(2 \times 10^{-3}\right.$ Torr $)$. Fig. 4 a shows a typical $\mathrm{I}_{\mathrm{SD}}-\mathrm{V}_{\mathrm{G}}$ curve with ambipolar behavior, which is similar to previous reported $\mathrm{CVD}$ grown graphene on $\mathrm{Ni}$ and $\mathrm{Cu}$ films ${ }^{18,28}$. For this particular test, we find the Dirac point to be $\sim 40 \mathrm{~V}$. This positive Dirac point suggests the presence of negative charges at ribbon- $\mathrm{SiO}_{2}$ interface ${ }^{42}$. This interface charge density can be calculated from the $\mathrm{SiO}_{2}$ capacitance and the value is found to be $2.9 \times 10^{12} \mathrm{~cm}^{-2}$ [see Supplementary Information for details]. From the slope of the source-drain variation, we extracted the room-temperature field effect mobility to be $655 \mathrm{~cm}^{2} \mathrm{~V}^{-1} \mathrm{~s}^{-1}$ for hole carriers. This high mobility value is three to five times higher than those of lithography patterned micro ribbons made from transfer-free Ni CVD graphene, which require further electron beam lithography and lift off procedures in order to obtain desired shape and structures for device applications ${ }^{18}$. The sheet resistance, $R_{\text {sheet }}$, is found to be $\sim 700 \Omega$ $-2100 \Omega$ per square, lower range than previously reported values (between $\sim 2000 \Omega$ per square to $\sim 5000 \Omega$ per square) of CVD graphene films directly grown on dielectric substrates ${ }^{18}$. In addition, unlike prior lithography patterned CVD graphene with marginal room-temperature current modulation ratio $\left(\mathrm{I}_{\text {on }} / \mathrm{I}_{\text {off }}\right)$ of $\sim 1.04-$ $1.4^{17,18,43}$, we found our directly grown graphene micro ribbons exhibit much higher current modulation of $\sim 3$ (Fig. $4 \mathrm{a}$ ), indicating strong potential for electrical gating applications.

In these two-point electrical transport measurements, the electrical contact resistance between the tungsten probe and graphene micro ribbons is non-negligible. The measured electrical resistance $\left(R_{\text {total }}\right)$ is the sum of contact resistance ( $R_{\text {contact }}$, which is the total contact resistance due to the source contact and the drain contact) and graphene micro ribbon channel resistance $\left(\mathrm{R}_{\text {channel }}\right)^{44}$. Since $\mathrm{R}_{\text {channel }}$ is linearly proportional to channel length to width ratio (L/ $\mathrm{W}$ ), therefore $\mathrm{R}_{\text {contact }}$ can be obtained from extrapolation at $\mathrm{L} / \mathrm{W}=0$. As shown in Fig. $4 b, R_{\text {total }}$ is plotted as a function of $L / W$ ratio under different gate biases (from $+60 \mathrm{~V}$ to $-60 \mathrm{~V}$ ). The extrapolation from the linear fit curves gives $R_{\text {contact }}=93 \pm 6 \mathrm{k} \Omega$. The slope of a linear fit curve represents the sheet resistance of the graphene micro ribbons $\left(R_{\text {sheet }}=\rho / t\right.$, where $\rho$ is the electrical resistivity, and $t$ is the thickness of the micro ribbon). As $\mathrm{V}_{\mathrm{G}}$ becomes more negative (from $+60 \mathrm{~V}$ to $-60 \mathrm{~V}$ ), $\mathrm{R}_{\text {sheet }}$ become smaller values (from $1850 \Omega$ per square to $1550 \Omega$ per square). This trend is consistent with the $V_{G}$ modulated conductance as shown in Fig. 4a.

\section{Discussion}

In order to explain the direct growth of graphene micro ribbons along the periphery of the pre-patterned Ni templates, we propose a mutual Ni dewetting and carbon diffusion mechanism. During the CVD annealing process, $\mathrm{C}_{2} \mathrm{H}_{4}$ decomposes and diffuses into Ni film through micrometer size Ni grain boundaries. Due to the low adhesion between $\mathrm{Ni}$ and $\mathrm{SiO}_{2}, \mathrm{Ni}$ dewets from the surface and forms into micrometer size domains near the periphery of the Ni film (Fig. 5a). The gaps between Ni micro domains promote the carbon diffusion on to the substrates. As the annealing and Ni evaporation proceeds, micro domains become smaller nano domains. More carbon source diffuses on to the substrate and carbon coverage increases with time. At the end of annealing process, a rapid cooling $\left(50^{\circ} \mathrm{C}\right.$ per minute $)$ is followed and drives carbon diffusion to form graphene ribbons. As a result of Ni dewetting, the thickness profile near the edge of the film shows distinctive change at the end of annealing process. Fig. 5b shows the convex profile of $\mathrm{Ni}$ edge recorded by a stylus profiler before the CVD annealing changes to a concave profile after the annealing. Ni dewets from the substrate near the edge and results in shrinkage of Ni. This further leads to the outer Ni piling up on top of the inner Ni because of steric crowding. Therefore, an abrupt increase of thickness is formed along the edges at the end of the annealing (as shown in by the red arrows in Fig. 5b). Fig. 5c shows a SEM micrograph of the region near the Ni film edge after the annealing process. A transition from continuous film to micro 

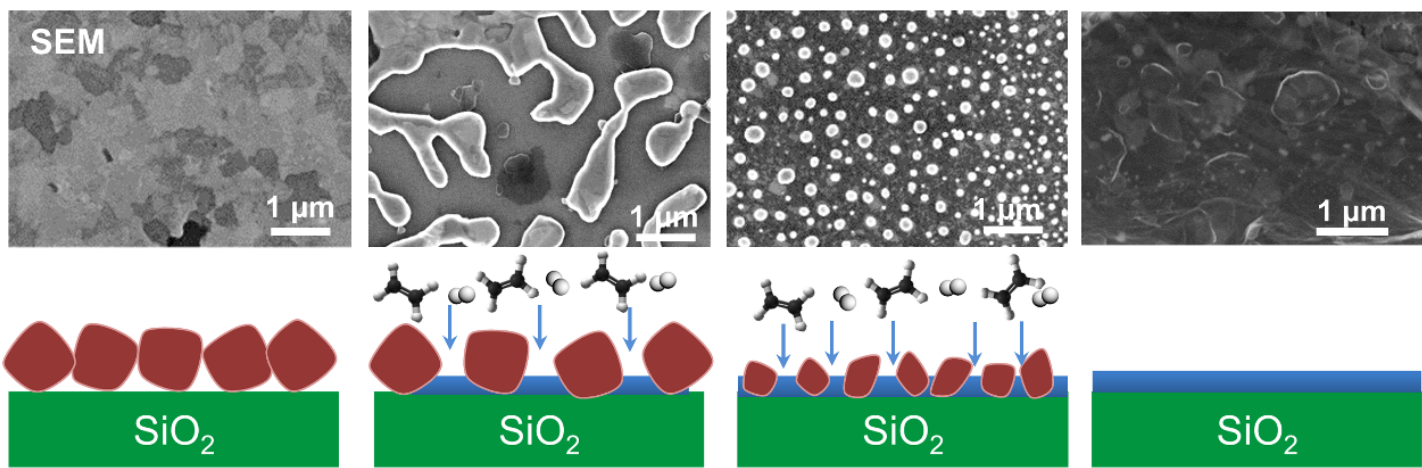

Continuous Ni film

Ni micro-grains

Ni nano-grains

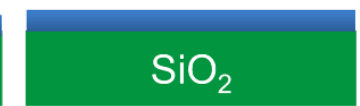

Graphene ribbons after $\mathrm{Ni}$ etching

b

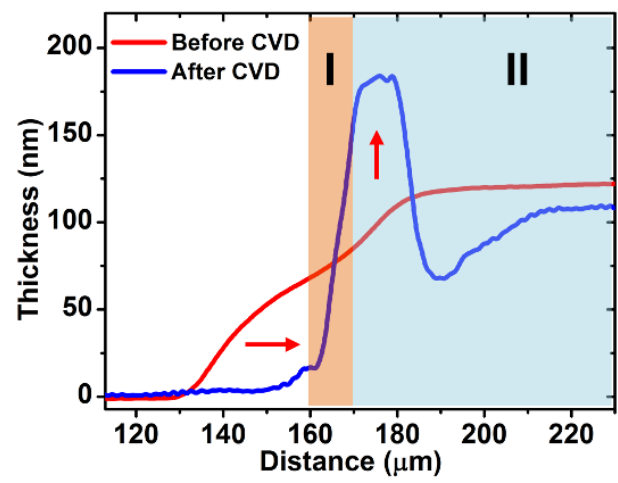

C

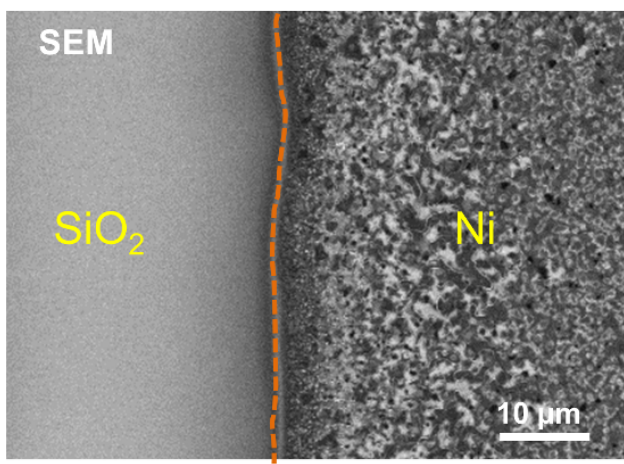

d

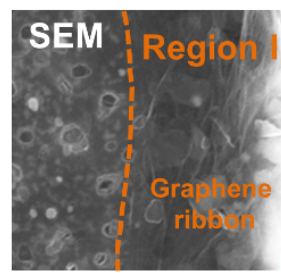

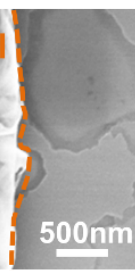
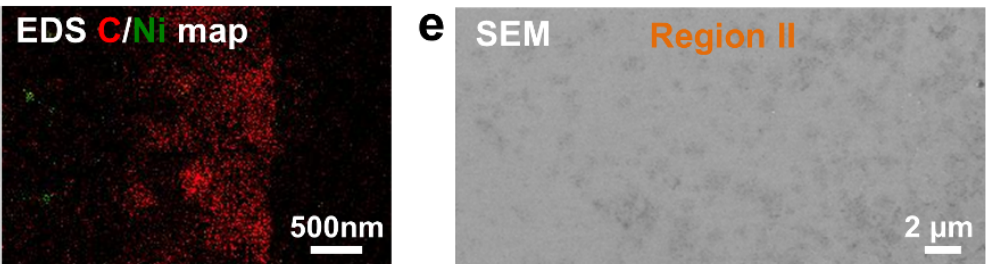

Figure $5 \mid$ Growth mechanism of the graphene micro ribbons. (a) A schematic illustrates the proposed growth mechanism to explain the direct growth of graphene micro ribbons along the periphery of Ni films. (b) The change of the thickness profile near the edge of a Ni film, before and after the

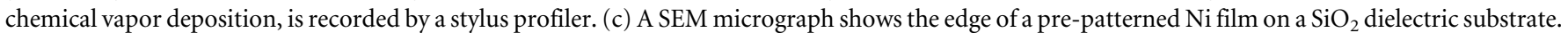
Before the CVD process, the profile shows a gradual decrease in thickness towards the edge. During the CVD deposition of graphene, Ni dewets from the $\mathrm{SiO}_{2}$ substrate surface. The outside of the film breaks into micrometer size grains. As dewetting proceeds, the Ni edge shrinks towards the inner part of the $\mathrm{Ni}$ film and piles up at the edge as shown by the red arrows in (b). Continuous graphene ribbons of a few micrometer widths are found in Region I. A representative graphene ribbon of $1.5 \mu \mathrm{m}$ is shown in a SEM micrograph and corresponding EDS map of carbon and $\mathrm{Ni}(\mathrm{d})$. On the other hand, in Region II where the diffusion of carbon source through Ni grains are much less efficient and dewetting of Ni is prohibited, given the short annealing time and low annealing temperature, only isolated micro domains of disordered carbon can be found (e).

domains and to nano domains is clearly seen. After etching away the $\mathrm{Ni}$ film, we find graphene micro ribbons to be a few micrometers wide. Here we refer this region as Region I (highlight in Fig. 5b). In this region, we performed an energy-dispersive spectroscopy (EDS) mapping (Fig. 5d). The mapping confirms the rich carbon content inside the graphene micro ribbon. Although the etching can thoroughly remove the Ni from the graphene micro ribbons, a few nanoparticles can found randomly under the graphene. These particles are identified as $\mathrm{Ni}$ as shown in the EDS map. They are very likely trapped under the graphene at the late stage of the CVD graphene growth. On the other hand, over the majority of $\mathrm{Ni}$ film area where the film thickness are uniform (Region II as defined in Fig. 5b), only isolated micro domains of disordered carbon can be found on the dielectric substrate (Fig. S3). This is due to the insufficient carbon diffusion as a result of the fast annealing at low temperature $\left(750^{\circ} \mathrm{C}\right.$ for 2-5 minutes), which we intentionally build into our method. Previous papers report continuous graphene films can be formed under the Ni using solid carbon source or gas phase carbon source with extended annealing time ( $\sim 20$ minutes) at elevated annealing temperature $\left(>1000^{\circ} \mathrm{C}\right)^{18,45}$. Because we are interested in growing graphene ribbons only along the periphery of the Ni film, we keep continuous graphene films from growing under the Ni film. As a result, the short annealing time and low annealing temperature slow down the graphene growth kinetics under the $\mathrm{Ni}$ in Region II (Fig. S4), whereas the Ni dewetting along the periphery promotes graphene growth in Region I. This figure of merit helps us to develop a methodology to grow graphene micro ribbons in a controllable manner.

The width of the graphene micro ribbons can be determined by the thickness profile of the Ni edges. As shown in Fig. 5b, a gradual variation of the thickness of Ni near the edges is formed as a result of deposition of prepatterned Ni. Since the melting temperature of $\mathrm{Ni}$ thin film is significantly lower than that of bulk materials and is strongly dependent on its thickness ${ }^{46}$, therefore a fraction of the edges separates from the continuous film and dewets into micron size or nanometer size domains during annealing process. We 
determine the graphene micro ribbons can grow in the area where the $\mathrm{Ni}$ thickness is below a threshold value $\sim 40 \mathrm{~nm}$ (corresponding to a $\sim 10 \mu \mathrm{m}$ wide area along the periphery of the $\mathrm{Ni}$ edges). Therefore, the width of the ribbons can be controlled by engineering the thickness profile near the Ni edges, i.e. the range of the area under the threshold thickness. Besides, since the melting temperature increase with film thickness ${ }^{46}$, raising the annealing temperature will increase the threshold thickess and hence increase the width of the graphene ribbons. In addition, the thickness of the majority of the prepatterned $\mathrm{Ni}$ has to be large enough so that its melting point is beyond the annealing temperature and graphene ribbons can only grow along its periphery.

We have demonstrated that continuous few-layer graphene micro ribbons up to few millimeters long can be grown directly on dielectric substrates. The dewetting of Ni from the dielectric substrates during the annealing process helps to facilitate the carbon diffusion on to the substrate surface and formation of graphene micro ribbons along the periphery of pre-patterned Ni template films. The short annealing time and low annealing temperature are the key factors to form continuous graphene micro ribbons near the Ni film edges, and no graphene films under the Ni film. The low annealing temperature is particularly useful to improve compatibility of graphene integration with microelectronic technologies and allows for energy efficiency.

Since the size, geometries, and locations of the micro ribbons are well defined by the pre-patterned $\mathrm{Ni}$ film, this present approach can be therefore scaled up to wafer size fabrication of graphene based devices. With better engineering of annealing temperature and annealing time, micro ribbons with better control in size, structural integrity, and number of layers can be envisioned. Efforts are currently underway to improve the electrical properties of the micro ribbons and their implementation into optoelectronic devices.

\section{Methods}

Direct growth of graphene micro ribbons on $\mathrm{SiO}_{2}$. We used $\mathrm{SiO}_{2}$ wafers as dielectric substrates for the direct growth of graphene micro ribbons. The wafers were purchased from Silicon Quest International $\left(300 \mathrm{~nm}\right.$ thick $\mathrm{SiO}_{2}$ layer thermally grown on $500 \mu \mathrm{m}$ thick $\mathrm{n}++$ type $\mathrm{Si}$ ). The $\mathrm{SiO}_{2}$ wafers were cleaned with piranha solution $\left(\mathrm{H}_{2} \mathrm{O}_{2} / \mathrm{H}_{2} \mathrm{SO}_{4}(1: 4)\right.$; Caution: piranha solutions are extremely dangerous and should be used with extreme caution) at $80^{\circ} \mathrm{C}$ for 30 minutes followed by intensive DI water rinsing before use. An MBruan thermal evaporator was used to deposit patterned $\mathrm{Ni}$ films on top of the $\mathrm{SiO}_{2}$ substrates. A shadow mask was placed in close contact with the $\mathrm{SiO}_{2}$ substrate before loading into the thermal evaporation chamber. During the thermal evaporation of Ni source (Sigma Aldrich, purity $>99.9 \%$ ), the deposition rate was kept at $\sim 2 \AA$ per second, and the chamber was kept at $10^{-6}$ mbar vacuum. The shapes and the sizes of Ni pattern were defined by the shadow mask. After the thermal evaporation is done, the thickness of Ni pattern was checked by a stylus profiler (Veeco Dektak 150 stylus surface profiler) to be $100 \mathrm{~nm}$.

For the CVD growth of graphene micro ribbons, the $\mathrm{SiO}_{2}$ substrate with pre-patterned Ni films was placed inside a quartz tube in a CVD furnace (Lindberg/ Blue Tube Furnace). After the furnace temperature was raised to $750^{\circ} \mathrm{C}$ within 30 minutes, a mixture of $\mathrm{H}_{2}$ and Ar flow (50 sccm: $150 \mathrm{sccm}$ ) was supplied into the quartz tube for 5 minutes. The pressure was kept at 10 Torr. This step ensured the removal of the native oxide layer of the Ni. Hydrocarbon source of graphene $\left(\mathrm{C}_{2} \mathrm{H}_{4}\right.$, $1 \mathrm{sccm}$ ) was then supplied into the quartz tube for annealing at $750^{\circ} \mathrm{C}$ with protective and reductive gas flow $\left(\mathrm{H}_{2}: \mathrm{Ar}=50 \mathrm{sccm}: 150 \mathrm{sccm}\right)$ under 10 Torr low pressure. The furnace is then allowed to cool down to room temperature at a rate of $50^{\circ} \mathrm{C}$ per minute, and the sample is then removed. The sample was rinsed in Ni etchant $(60 \%$ $\mathrm{H}_{2} \mathrm{O}, 35 \% \mathrm{FeCl}_{3}, 5 \% \mathrm{HCl}$ ). The Ni films together with the graphene films grown on top surface were subsequently etched away, leaving only graphene micro ribbons on the dielectric substrate.

Spectroscopic and microscopic characterization. The number of graphene layers and structural quality of as-grown graphene micro ribbon was examined by confocal Raman spectroscopy and microscopy (HORIBA Jobin Yvon LabRAM ARAMIS micro Raman system, $532 \mathrm{~nm}$ excitation laser, Olympus $100 \times$ NA 0.9 long working distance objective, mapping spatial resolution $\sim 1 \mu \mathrm{m}$ ). The morphology of the graphene micro ribbons was further characterized by scanning electron microscope (Zeiss analytical FE-SEM Ultra55) and atomic force microscope (Veeco Dimension with Nanoscope $\mathrm{V}$ controller). High resolution tapping mode images were acquired with ultra-sharp silicon tips (Nanosensors PPP-NCHR probes, resonance frequency $330 \mathrm{kHz}$, nominal spring constant $42 \mathrm{~N} / \mathrm{m}$ ). The AFM image data was analyzed by SPIP software (Image Metrology A/S). Energy-dispersive spectroscopy (EDS) mapping was acquired using the EDAX detector attached to the Zeiss analytical FE-SEM Ultra55.
Electrical measurements. The two-point electrical tests (I-V characteristics) were measured with a semiconductor parameter analyzer (Agilent 4155C) at room temperature. The samples were placed into a probe station (Lakeshore CPX-HF) under vacuum condition $\left(2 \times 10^{-3}\right.$ Torr). Two tungsten microprobes (type: ZN50R-25-W, $25 \mu \mathrm{m}$ tip radius) were used as source and drain electrodes, and placed directly on top of several segments of graphene micro ribbons. The highly doped $\mathrm{Si}$ $\left(\mathrm{n}++\right.$ type) under the $\mathrm{SiO}_{2}$ dielectric layer was used as the gate electrode. The source-drain current $\left(\mathrm{I}_{\mathrm{SD}}\right)$ was modulated by applying a sweeping back-gate voltage $\left(\mathrm{V}_{\mathrm{G}}\right)$.

1. Novoselov, K. S. et al. A roadmap for graphene. Nature 490, 192-200 (2012).

2. Geim, A. K. \& Novoselov, K. S. The rise of graphene. Nat. Mater. 6, 183-191 (2007).

3. Chen, J. H., Jang, C., Xiao, S. D., Ishigami, M. \& Fuhrer, M. S. Intrinsic and extrinsic performance limits of graphene devices on $\mathrm{SiO}_{2}$. Nat. Nanotechnol. 3, 206-209 (2008).

4. Lin, Y. M. et al. 100-GHz transistors from wafer-scale epitaxial graphene. Science 327, 662-662 (2010).

5. Wang, F. et al. Gate-variable optical transitions in graphene. Science 320, 206-209 (2008).

6. Ju, L. et al. Graphene plasmonics for tunable terahertz metamaterials. Nat. Nanotechnol. 6, 630-634 (2011).

7. Chen, X. Y. et al. Fully Integrated Graphene and Carbon Nanotube Interconnects for Gigahertz High-Speed CMOS Electronics. IEEE T. Electron. Dev. 57, 3137-3143 (2010).

8. Novoselov, K. S. et al. Two-dimensional atomic crystals. P. Natl. Acad. Sci. USA 102, 10451-10453 (2005)

9. Novoselov, K. S. et al. Electric field effect in atomically thin carbon films. Science 306, 666-669 (2004).

10. Stankovich, S. et al. Graphene-based composite materials. Nature 442, 282-286 (2006).

11. Eda, G., Fanchini, G. \& Chhowalla, M. Large-area ultrathin films of reduced graphene oxide as a transparent and flexible electronic material. Nat. Nanotechnol. 3, 270-274 (2008).

12. Hernandez, Y., Lotya, M., Rickard, D., Bergin, S. D. \& Coleman, J. N. Measurement of multicomponent solubility parameters for graphene facilitates solvent discovery. Langmuir 26, 3208-3213 (2010).

13. de Heer, W. A. et al. Epitaxial graphene. Solid State Commun. 143, 92-100 (2007)

14. Sprinkle, M. et al. Scalable templated growth of graphene nanoribbons on SiC. Nat. Nanotechnol. 5, 727-731 (2010).

15. Wei, Z. Q. et al. Nanoscale tunable reduction of graphene oxide for graphene electronics. Science 328, 1373-1376 (2010).

16. Kim, K. S. et al. Large-scale pattern growth of graphene films for stretchable transparent electrodes. Nature 457, 706-710 (2009).

17. Reina, A. et al. Large area, few-layer graphene films on arbitrary substrates by chemical vapor deposition. Nano Lett. 9, 30-35 (2009).

18. Yan, Z. et al. Growth of bilayer graphene on insulating substrates. ACS Nano $\mathbf{5}$, 8187-8192 (2011).

19. Li, X. S. et al. Large-area synthesis of high-quality and uniform graphene films on copper foils. Science 324, 1312-1314 (2009).

20. Ismach, A. et al. Direct chemical vapor deposition of graphene on dielectric surfaces. Nano Lett. 10, 1542-1548 (2010).

21. Kwon, S. Y. et al. Growth of semiconducting graphene on palladium. Nano Lett. 9 , 3985-3990 (2009).

22. Sutter, P. W., Flege, J. I. \& Sutter, E. A. Epitaxial graphene on ruthenium. Nat. Mater. 7, 406-411 (2008).

23. Coraux, J., N'Diaye, A. T., Busse, C. \& Michely, T. Structural coherency of graphene on $\operatorname{Ir}(111)$. Nano Lett. 8, 565-570 (2008).

24. Vaari, J., Lahtinen, J. \& Hautojarvi, P. The adsorption and decomposition of acetylene on clean and K-covered Co(0001). Catal. Lett. 44, 43-49 (1997).

25. Ueta, H. et al. Highly oriented monolayer graphite formation on $\mathrm{Pt}(111)$ by a supersonic methane beam. Surf. Sci. 560, 183-190 (2004).

26. Regan, W. et al. A direct transfer of layer-area graphene. Appl. Phys. Lett. 96, 113102 (2010).

27. Aleman, B. et al. Transfer-free batch fabrication of large-area suspended graphene membranes. ACS Nano 4, 4762-4768 (2010).

28. Bae, S. et al. Roll-to-roll production of 30-inch graphene films for transparent electrodes. Nat. Nanotechnol. 5, 574-578 (2010).

29. Srivastava, A. et al. Novel liquid precursor-based facile synthesis of large-area continuous, single, and few-layer graphene films. Chem. Mater. 22, 3457-3461 (2010).

30. Venugopal, A. et al. Effective mobility of single-layer graphene transistors as a function of channel dimensions. J. Appl. Phys. 109, 104511 (2011).

31. Jiao, L. Y., Zhang, L., Wang, X. R., Diankov, G. \& Dai, H. J. Narrow graphene nanoribbons from carbon nanotubes. Nature 458, 877-880 (2009).

32. Cai, J. M. et al. Atomically precise bottom-up fabrication of graphene nanoribbons. Nature 466, 470-473 (2010).

33. Lee, K. J., Chandrakasan, A. P. \& Kong, J. Breakdown current density of CVD-grown multilayer graphene interconnects. IEEE Electr. Device. L. 32, 557-559 (2011) 
34. Malard, L. M., Pimenta, M. A., Dresselhaus, G. \& Dresselhaus, M. S. Raman spectroscopy in graphene. Phys. Rep. 473, 51-87 (2009).

35. Zhu, Y. W. et al. Graphene and graphene oxide: synthesis, properties, and applications. Adv. Mater. 22, 3906-3924 (2010).

36. Tao, L. et al. Synthesis of high quality monolayer graphene at reduced temperature on hydrogen-enriched evaporated copper (111) Films. ACS Nano 6, 2319-2325 (2012).

37. Su, C. Y. et al. Direct formation of wafer scale graphene thin layers on insulating substrates by chemical vapor deposition. Nano Lett. 11, 3612-3616 (2011).

38. Mohanty, N. et al. Nanotomy-based production of transferable and dispersible graphene nanostructures of controlled shape and size. Nat. Commun. 3, 844 (2012)

39. Chen, Z. P. et al. Three-dimensional flexible and conductive interconnected graphene networks grown by chemical vapour deposition. Nat. Mater. 10, 424-428 (2011).

40. Chae, S. J. et al. Synthesis of large-area graphene layers on poly-nickel substrate by chemical vapor deposition: wrinkle formation. Adv. Mater. 21, 2328-2333 (2009).

41. Yoshizawa, K., Yumura, T., Yamabe, T. \& Bandow, S. The role of orbital interactions in determining the interlayer spacing in graphite slabs. J. Am. Chem. Soc. 122, 11871-11875 (2000).

42. Shishir, R. S., Chen, F., Xia, J., Tao, N. J. \& Ferry, D. K. Room temperature carrier transport in graphene. J. Comput. Electron. 8, 43-50 (2009).

43. Kwak, J. et al. Near room-temperature synthesis of transfer-free graphene films. Nat. Commun. 3, 645 (2012).

44. Jo, G. et al. Channel-length and gate-bias dependence of contact resistance and mobility for $\mathrm{In}_{2} \mathrm{O}_{3}$ nanowire field effect transistors. J. Appl. Phys. 102, 084508 (2007).

45. Peng, Z. W., Yan, Z., Sun, Z. Z. \& Tour, J. M. Direct growth of bilayer graphene on $\mathrm{SiO}_{2}$ substrates by carbon diffusion through nickel. ACS Nano 5, 8241-8247 (2011).
46. Gromov, D. G. et al. Fluctuation phenomena in nanoscale nickel films near the melting point. Semiconductors 45, 1689-1693 (2011).

\section{Acknowledgments}

This work was supported by the Office of Science, Office of Basic Energy Sciences of the U.S. Department of Energy (No. DE-AC02-05CH11231) and National Natural Science Foundation $(61025021,60936002,51072089,61020106006)$ and National Key Project of Science \& Technology (2009ZX02023-001-3, 2011ZX02403-002) of China. H.T. thanks additional support from the Ministry of Education Scholarship of China. Lawrence Berkeley National Laboratory and Tsinghua University contributed equally to this work.

\section{Author contributions}

T.-L.R. and Y.Z. proposed and supervised the project. D.W. and H.T. designed and carried out the experiments. D.W. and H.T. analyzed data. All the authors participated in the discussion and writing of the manuscript.

\section{Additional information}

Supplementary information accompanies this paper at http://www.nature.com/ scientificreports

Competing financial interests: The authors declare no competing financial interests License: This work is licensed under a Creative Commons Attribution-NonCommercial-ShareALike 3.0 Unported License. To view a copy of this license, visit http://creativecommons.org/licenses/by-nc-sa/3.0/

How to cite this article: Wang, D. et al. Scalable and Direct Growth of Graphene Micro Ribbons on Dielectric Substrates. Sci. Rep. 3, 1348; DOI:10.1038/srep01348 (2013) 